\title{
Rapamycin prevents the mutant huntingtin- suppressed GLT-1 expression in cultured astrocytes
}

\author{
Lei-lei CHEN ${ }^{1}$, Jun-chao $\mathrm{WU}^{1}$, Lin-hui WANG ${ }^{1}$, Jin WANG ${ }^{1}$, Zheng-hong $\mathrm{QIN}^{1}$, Marian DIFIGLIA ${ }^{2}$, Fang LIN $^{1, \text { * }}$ \\ ${ }^{1}$ Laboratory of Aging and Nervous Diseases and Department of Pharmacology, Soochow University School of Pharmaceutical Science, \\ Suzhou 215123, China; ' 2Laboratory of Cellular Neurobiology, Massachusetts General Hospital and Harvard Medical School, Charles- \\ town, MA 02129, USA
}

\begin{abstract}
Aim: To investigate the effects of rapamycin on glutamate uptake in cultured rat astrocytes expressing $\mathrm{N}$-terminal 552 residues of mutant huntingtin (Htt-552).

Methods: Primary astrocyte cultures were prepared from the cortex of postnatal rat pups. An astrocytes model of Huntington's disease was established using the astrocytes infected with adenovirus carrying coden gene of N-terminal 552 residues of Huntingtin. The protein levels of glutamate transporters GLT-1 and GLAST, the autophagic marker microtubule-associated protein 1A/1B-light chain 3 (LC3) and the autophagy substrate p62 in the astrocytes were examined using Western blotting. The mRNA expression levels of GLT-1 and GLAST in the astrocytes were determined using Real-time PCR. $\left[{ }^{3} \mathrm{H}\right]$ glutamate uptake by the astrocytes was measured with liquid scintillation counting.

Results: The expression of mutant Htt-552 in the astrocytes significantly decreased both the mRNA and protein levels of GLT-1 but not those of GLAST. Furthermore, Htt-552 significantly reduced $\left[{ }^{3} \mathrm{H}\right]$ glutamate uptake by the astrocytes. Treatment with the autophagy inhibitor 3-MA (10 mmol/L) significantly increased the accumulation of mutant Htt-552, and reduced the expression of GLT-1 and $\left[{ }^{3} \mathrm{H}\right]$ glutamate uptake in the astrocytes. Treatment with the autophagy stimulator rapamycin $(0.2 \mathrm{mg} / \mathrm{mL})$ significantly reduced the accumulation of mutant $\mathrm{Htt}-552$, and reversed the changes in GLT-1 expression and $\left[{ }^{3} \mathrm{H}\right]$ glutamate uptake in the astrocytes.

Conclusion: Rapamcin, an autophagy stimulator, can prevent the suppression of GLT-1 expression and glutamate uptake by mutant Htt552 in cultured astrocytes.
\end{abstract}

Keywords: Huntington's disease; huntingtin-552; GLT-1; glutamate uptake; autophagy; rapamycin; 3-MA

Acta Pharmacologica Sinica (2012) 33: 385-392; doi: 10.1038/aps.2011.162; published online 23 Jan 2012

\section{Introduction}

Huntington's disease (HD) is a neurological disorder that is caused by an expansion of a polyglutamine tract in the protein huntingtin (Htt). Although mutant huntingtin is widely expressed in neuronal and non-neuronal cells, it preferentially accumulates in striatal neurons and causes neurodegeneration in the brain ${ }^{[1]}$. This phenomenon has led to extensive studies of mutant huntingtin on neurons. Later studies have found that in a neuron-glia co-culture system, wild-type glial cells protect neurons against neurotoxicity caused by mutant Htt, whereas glial cells expressing mutant Htt increased neuronal vulnerability ${ }^{[2]}$. These studies indicate that cell-cell interactions between neurons and glial cells play an important role in HD pathology ${ }^{[3,4]}$. In addition, mutant Htt expressed in glial cells could exacerbate neurological symptoms in HD trans-

\footnotetext{
* To whom correspondence should be addressed.

E-mail bluestonelin@hotmail.com

Received 2011-07-04 Accepted 2011-11-03
}

genic mice, so the role of glial cells in HD neuropathology should not be neglected ${ }^{[5]}$.

Recently, some studies have proven that the selective degeneration of striatal neurons is relevant to dysfunctional glial protective mechanisms in HD pathology ${ }^{[2,6]}$. On the membranes of astrocytes, which are the major subtype of glial cells, there are two types of glutamate transporters (GLT-1 and GLAST) that do most of the work in clearing extracellular excitatory neurotransmitters ${ }^{[7,8]}$. It has been shown that small fragments of N-terminal mutant Htt (such as N-208, N-171), which were reported to be more pathogenic than full-length mutant Htt, caused decreased expression of GLT-1 ${ }^{[2,5]}$. However, these fragments had been cut occasionally and might not exist in physiological and pathological conditions, so they could not simulate the condition of HD patients completely. In this study, a termination codon was inserted into Htt at the N-terminal 552 amino acid of caspase2/3 and generated a truncated Htt fragment 1-552 aa (Htt-552). Mutant Htt-552 had been found in the brains of HD patients, which allows us 
to achieve a similar pathological condition of HD. Moreover, we intended to find out if mutant Htt-552 expressed in cultured astrocytes had the same effects as N-208 and N-171.

It was reported that the decreased glutamate uptake in astrocytes was mainly caused by the decreased expression of GLT-1, which has a gene promoter that carries multiple Sp1 binding sites ${ }^{[9]}$. The transcription of GLT-1 was Sp1dependent, and it had been reported that mutant Htt bound more Sp1 and reduced Sp1-mediated GLT-1 expression in astrocytes. This could lead to defective glial glutamate uptake and increased neuronal excitotoxicity ${ }^{[10]}$. Therefore, we want to know if the expression of GLT-1 would return to a normal level after mutant Htt had been cleared.

Autophagy is a major degradation pathway for misfolded proteins, especially for long-lived proteins. It was reported that enhancing autophagy with rapamycin treatment increased mutant huntingtin clearance and decreased the levels of soluble proteins and aggregates in HD cell models ${ }^{[11]}$. In contrast, inhibition of autophagy during autophagosome formation by 3-MA decreased mutant huntingtin clearance and increased the levels of soluble and aggregated mutant huntingtin ${ }^{[12]}$. In our previous studies, we also found that activated autophagy could clear mutant Htt effectively ${ }^{[13]}$. Therefore, in this study, we used rapamycin, an autophagy activator, to enhance autophagy in astrocytes and to investigate if the expression of GLT-1 could be returned to its initial level.

\section{Materials and methods Reagents}

Huntingtin antibody (1:2000, Cat.MAB2166, Chemicon, Billerica, MA, USA); $\beta$-actin antibody (1:5000, Cat.A5441, Sigma, St Louis, MO, USA); LC3 antibody (1:1000, Cat.Ab62721, Abcam, University of Cambridge, UK); p62 antibody (1:2000, Cat.PW9860, Enzo Life Science, Lausen, CH, USA); GFAP (1:2000, Cat.c9205, Sigma, St Louis, MO, USA); DAPI (1:10000, Cat. D9564, Sigma, St Louis, MO, USA); GLT-1 (1:3000, Cat. ab58571, Abcam, University of Cambridge, UK); GLAST (1:5000, Cat.ab416, Abcam, University of Cambridge, UK); Cy3-conjugated anti-mouse IgG and HRP-conjugated antimouse IgG (1:5000, Cat 715-165-150 and Cat 715-035-1500, Jackson ImmunoResearch, West Grove, PA, USA); rapamycin (Cat. R0395, Sigma, St Louis, MO, USA); 3-MA (3-methyladenine, Cat. M9281, Sigma, St Louis, MO, USA); DHK (dihydrokainate, Cat.D1064, Sigma, St Louis, MO, USA); and $\left[{ }^{3} \mathrm{H}\right]$ glutamate (Cat.NET1082250UC, Perkin Elmer, Waltham, MA, USA) were used in this study.

\section{Primary astrocyte culture}

Primary astrocyte cultures were prepared from the cortex of 1- to 2-day-old postnatal SD rat pups as described in a previous study ${ }^{[14]}$. The ethical committee of Soochow University approved this study. Microglial cells were dissociated from the culture by shaking cultured glial cells. An immunoassay with antibodies against GFAP was used to identify the purity of the astrocytes.

\section{Western blot analysis}

Astrocytes were harvested $24 \mathrm{~h}, 48 \mathrm{~h}$, or $72 \mathrm{~h}$ after infection. The boiled samples (each containing 10-20 $\mu \mathrm{g}$ of protein) were subjected to SDS-PAGE on a $10 \%$ acrylamide gel and transferred to nitrocellulose membranes (Bio-Rad, Hercules, CA, USA). The membranes were blocked for $1 \mathrm{~h}$ in TBST containing $5 \%$ non-fat milk. The membranes were then incubated with mouse anti-Htt monoclonal antibody 2166 (1:2000, Chemicon) at $4{ }^{\circ} \mathrm{C}$ overnight, incubated with horseradish peroxidase-conjugated donkey anti-mouse IgG secondary antibody (Sigma) at a dilution of 1:5000 for $2 \mathrm{~h}$ and finally visualized with an enhanced chemiluminescence (ECL) kit (Shanghai Sangon Biological Engineering Technology, Shanghai, China) according to the manufacturer's protocol.

\section{Real-time PCR}

The real-time PCR experiments were performed according to the protocol of the Real-time PCR kit (Cat.DRR041A, TAKARA). The primers were as follows: GAPDH: forward primer: $5^{\prime}$-gacaactttggcatcgtgga-3', reverse primer: $5^{\prime}$-atgcagggatgatgttctgg-3'; GLAST: forward primer: $5^{\prime}$-gectttgtgctactcaccgtca-3', reverse primer: $5^{\prime}$-ctgcagcatccgcatcaga-3'; GLT-1: forward primer: $5^{\prime}$-gcaggtggaagtgcgcatgcac- $3^{\prime}$, reverse primer: $5^{\prime}$-cacatactgctcccaggatgaca- $3^{\prime}$.

\section{Glutamate uptake assay}

After being infected with Ad-Htt-552-18Q, Ad-Htt-552-100Q, or Ad-null, the astrocytes were washed with normal saline. Half of the cells were pre-incubated with $1 \mathrm{mmol} / \mathrm{L}$ dihydrokainate (DHK; Sigma Aldrich) for $1 \mathrm{~h}$ at $37^{\circ} \mathrm{C}$, and the other half were pre-incubated without DHK treatment. After pre-incubation, $\left[{ }^{3} \mathrm{H}\right]$ glutamate at a concentration of $25 \mathrm{nmol} / \mathrm{L}$ was added into the solution and incubated for $15 \mathrm{~min}$. The incubation was terminated by rapidly removing the solution, and the astrocytes were washed with $4 \mathrm{~mL}$ of ice-cold normal saline three times. The astrocytes were lysed in $0.3 \% \mathrm{NaOH}$ with sonication, and the radioactivity was determined by using a liquid scintillation counter (Perkin Elmer, Waltham, MA, USA). The protein concentration was measured to normalize the scintillation counting results. The astrocytes preincubated with $10 \mathrm{mmol} / \mathrm{L}$ of unlabeled glutamate served as a control to obtain a background value. The difference between the DHK-treated and non-treated samples was obtained and reflected GLT-1-specific glutamate uptake $(\mathrm{nmol} \cdot \mathrm{mg}$ pro$\left.\operatorname{tein}^{-1} \cdot 15 \mathrm{~min}^{-1}\right)$.

\section{Statistical analysis}

Statistical significance was assessed by using one-way ANOVA with the Newman-Keuls multiple comparison test. Calculations were performed using Sigma Plot 4.11 and Prism (version 4) software. Statistical significance was considered when $P<0.05$.

\section{Results}

Expression of Htt-552 in cultured primary astrocytes

The purity of astrocytes reached approximately $98 \%$ and met 
A
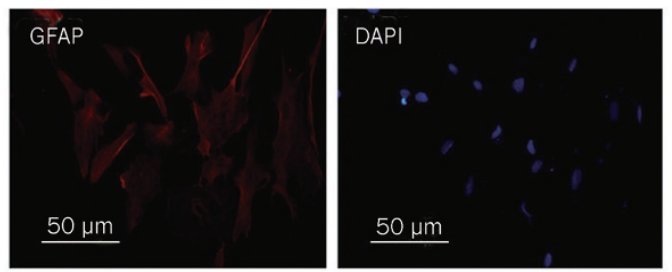

$\mathrm{B}_{180}$
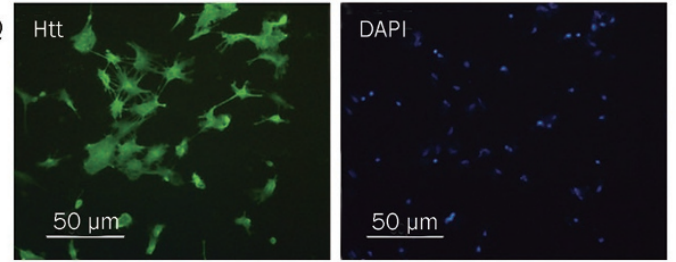

$100 Q$
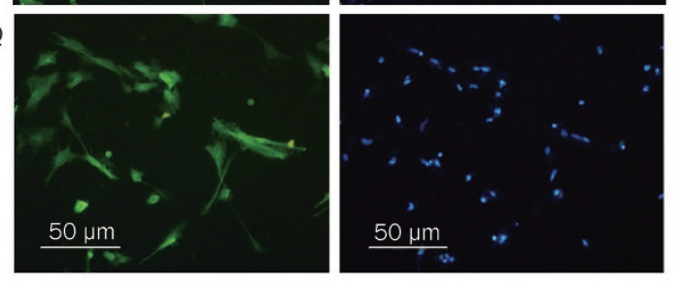

C

$$
\text { Con } \frac{\text { Null }}{24 h 48 h 72 h} \frac{18 Q}{24 h 48 h 72 h} \frac{100 Q}{24 h 48 h 72 h}
$$

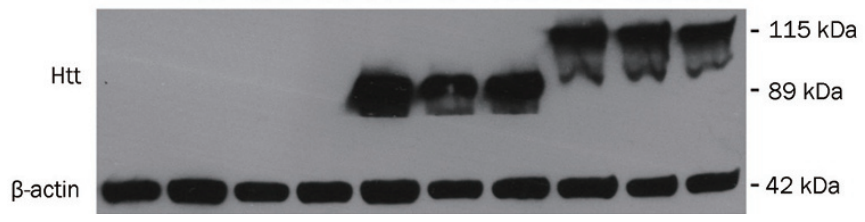

Figure 1. Expression of Htt-552 in cultured astrocytes. (A) Astrocytes were collected from 1- to 2-day-old postnatal rats and purified to the third generation. GFAP is a marker of astrocytes. DAPI stains cell nuclei. Immunofluorescence labeling showed more than 98\% of the GFAPpositive (red) astrocytes. (B) Immunofluorescent images of cultured glial cells that were infected with Ad-Htt-552-18Q (wild type Htt) or Ad-Htt-552$100 \mathrm{Q}$ (mutant $\mathrm{Htt}$ ) for $24 \mathrm{~h}$ show that the ratio of Htt-positive cells to DAPIpositive cells is almost $80 \%$. (C) Western blotting analysis of Htt-552 in cultured astrocytes 24,48 , and $72 \mathrm{~h}$ after infection, showing persistent expression of wild type $\mathrm{Htt}$ and mutant $\mathrm{Htt}$.

our study's needs (Figure 1A). The astrocytes were harvested after being infected with Ad-Htt-552-18Q or Ad-Htt-552-100Q for $24 \mathrm{~h}, 48 \mathrm{~h}$, or $72 \mathrm{~h}$. Then immunohistochemistry staining and Western blot analysis were performed to identify the expression of wild-type $\mathrm{Htt}(\mathrm{Htt}-552-18 \mathrm{Q})$ and mutant $\mathrm{Htt}$ (Htt-552-100Q) in the astrocytes. The proper ratio of infection is very important to this study. We found that the adenoviral tier, which resulted in $80 \%$ of the cultured astrocytes expressing mutant $\mathrm{Htt}$, was thought to be ideal (Figure 1B). Western blot analysis showed that persistent stable expression of $\mathrm{Htt}$ in the astrocytes lasted for at least $72 \mathrm{~h}$ after infection (Figure 1C).

Decreased expression of GLT-1 and glutamate uptake in astrocytes

Western blot analysis revealed decreased expression of GLT-1 in astrocytes expressing mutant Htt (Ad-Htt-552-100Q), and the decreased expression of GLT-1 appeared to correlate with the time-dependent expression of mutant $\mathrm{Htt}$ in astrocytes (Figure 2A). However, the level of expression of GLAST appeared to be variable in astrocytes but not significantly decreased (Figure 2C). Real-time PCR confirmed that GLT-1 transcripts were significantly reduced in astrocytes expressing mutant Htt for 48 or $72 \mathrm{~h}$ (Figure 2B), while GLAST transcripts showed no significant changes (Figure 2D). There was no obvious effect on either GLT-1 mRNA or GLT-1 proteins by wild-type Htt expression (Ad-Htt-552-18Q).

An interesting question is whether fewer glutamate transporters could alter glutamate uptake in astrocytes. We decided to infect astrocytes with Ad-null, Ad-Htt-552-18Q or Ad-Htt$100 \mathrm{Q}$ for $72 \mathrm{~h}$ and then measure their uptake of $\left[{ }^{3} \mathrm{H}\right]$ glutamate as described in the methods section. The result showed significantly decreased glutamate uptake in astrocytes expressing mutant $\mathrm{Htt}$, which was closely correlated with altered expression of GLT-1 (Figure 2E). It was reported that DHK could specifically inhibit the activity of GLT- 1 in astrocytes ${ }^{[15]}$. After infecting the astrocytes with Ad-null, Ad-Htt-552-18Q or AdHtt-552-100Q for $72 \mathrm{~h}$, we pre-incubated the astrocytes with 1 $\mathrm{mmol} / \mathrm{L}$ DHK for $1 \mathrm{~h}$ at $37^{\circ} \mathrm{C}$, and the control was pre-incubated without DHK treatment. The difference between the astrocytes that received the DHK treatment and those that did not received the DHK treatment revealed the specific contribution of GLT-1 to transporting $\left[{ }^{3} \mathrm{H}\right]$ glutamate. Consistent with the decreased expression of GLT-1, GLT-1-specific glutamate uptake was more significantly decreased in astrocytes expressing mutant $\mathrm{Htt}$, demonstrating a close association between GLT-1 expression and glutamate uptake in astrocytes.

\section{Enhancing Htt clearance by activation of autophagy}

After transfection of cells with Ad-Htt-552-18Q or Ad-Htt$552-100 \mathrm{Q}$ for $48 \mathrm{~h}$, the cells were treated with rapamycin $(0.2$ $\mu \mathrm{g} / \mathrm{mL})$, an activator of autophagy, or 3-MA (10 mmol/L), an inhibitor of autophagy. The astrocytes were then harvested for Western blot analysis $24 \mathrm{~h}$ later. The microtubuleassociated protein 1A/1B-light chain 3 (LC3) is thought to be an autophagic marker ${ }^{[16]}$. An increased ratio of LC3II to LC3I was observed when the astrocytes were treated with rapamycin. On the contrary, when infected astrocytes were treated with 3-MA, the expression of LC3II did not seem to change significantly. But compared with the control, the expression of LC3I increased, so the rate of LC3II/LC3I decreased. These results suggested that autophagy was activated by rapamycin and inhibited by 3-MA (Figure 3A). p62/SQSTM1 is an ubiquitin- and LC3-binding protein that could be degraded by autophagy and is thought to be a substrate of autophagy ${ }^{[17]}$. We found that the amount of p62 was decreased when cells were treated with rapamycin (100Q-rap vs 100Q) (Figure $3 \mathrm{~B})$, while the amount of p62 was increased when cells were treated with 3-MA (100Q-3-MA vs 100Q) (Figure 3C). These results confirmed that autophagy was enhanced by rapamycin and inhibited by 3-MA.

Furthermore, we detected the protein levels of Htt. Western 
A

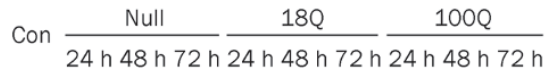

GLT-1

$\beta$-actin
C
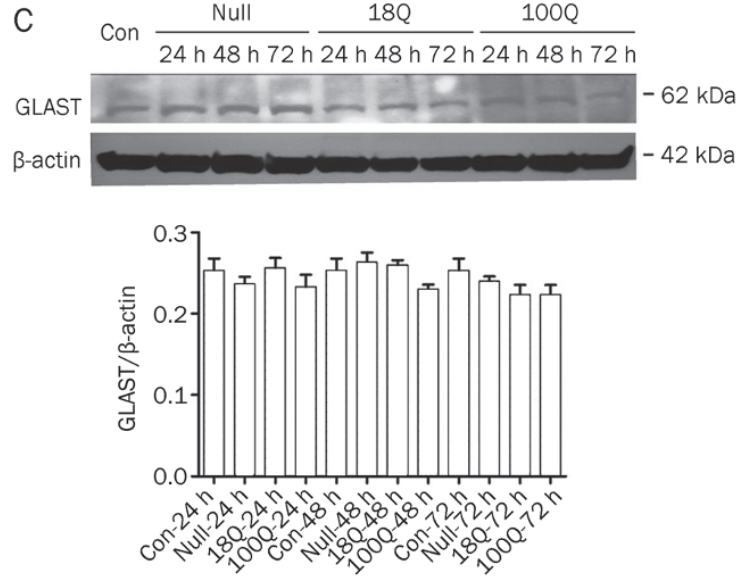

D
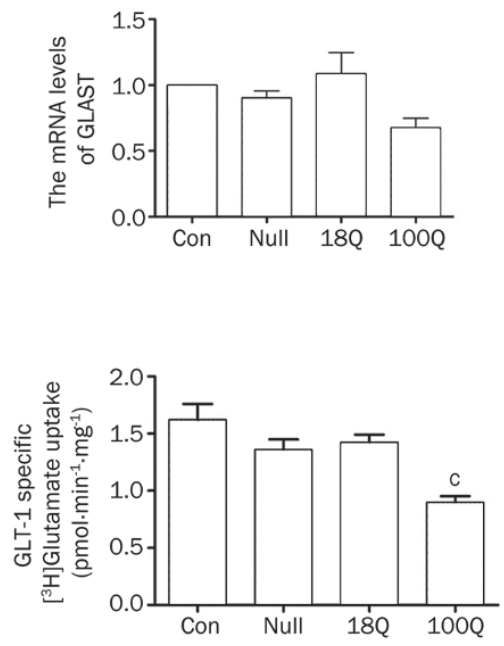

Figure 2. Decreased expression of GLT-1 and glutamate uptake in astrocytes. Cultured astrocytes were harvested after expression of Htt-552 for 24,48 , or $72 \mathrm{~h}$. The results are shown as means \pm SD $(n=3)$. (A) Western blotting analysis was performed, showing decreased expression of GLT-1 in astrocytes expressing mutant Htt. ${ }^{\circ} P<0.01,100 \mathrm{Q}-24 \mathrm{~h}$ vs (con-24 h, null-24 h, 18Q-24 h); ${ }^{\mathrm{f}} \mathrm{P}<0.01,100 \mathrm{Q}-48 \mathrm{~h}$ vs (con-48 h, null-48 h, $18 \mathrm{Q}-$ 48 h); ' $P<0.01,100 Q-72$ h vs (con-72 h, null-72 h, 18Q-72 h). (B) The total RNA in astrocytes was extracted after expression of Htt-552 for 72 h. Realtime PCR showed decreased GLT-1 transcription in astrocytes expressing mutant Htt for $72 \mathrm{~h}$ ('P<0.01 compared with con, null and 18Q). (C) Western blotting analysis showed no significant difference in the expression of GLAST in astrocytes after expression of mutant and wild-type Htt-552. (D) The total RNA in astrocytes was extracted after expression of Htt-552 for $72 \mathrm{~h}$. Real-time PCR showed no significant difference in GLAST mRNA levels in astrocytes. (E) Decreased glutamate uptake by astrocytes expressing mutant Htt. GLT-1-specific glutamate uptake was significantly decreased in astrocytes expressing mutant $\mathrm{Htt}\left({ }^{\mathrm{C}} P<0.01,100 \mathrm{Q}\right.$ vs cont, null, 18Q), as well as the total uptake level ( ${ }^{\mathrm{C}} P<0.01,100 \mathrm{Q}$ vs cont, null, 18Q). After expression of Htt-552 for $72 \mathrm{~h}$, the astrocytes were pre-incubated with $1 \mathrm{mmol} / \mathrm{L}$ DHK (specific inhibitor of GLT-1) for $1 \mathrm{~h}$ at $37^{\circ} \mathrm{C}$, and GLT-1-specific glutamate uptake was determined (one-way ANOVA with Newman-Keuls multiple comparison test).

blot analysis showed a significant reduction of mutant $\mathrm{Htt}$ when autophagy was stimulated (100Q-rap vs 100Q) (Figure $3 \mathrm{~B})$, and a significant accumulation of mutant $\mathrm{Htt}$ was observed when autophagy was inhibited (100Q-3-MA vs 100Q) (Figure 3C).

\section{Recovery of GLT-1 expression and function by rapamycin}

It has been reported that the decreased expression of GLT-1 had been mainly caused by mutant $\mathrm{Htt}$. As the mutant $\mathrm{Htt}$ is cleared by enhanced autophagy, could the expression of GLT-1 be resumed? In this study, we detected the expression of GLT-1 and glutamate uptake by astrocytes after treatment with rapamycin. Western blot analysis showed a recovery of GLT-1 levels in astrocytes when autophagy was activated by rapamycin (Figure 4A). With the treatment of 3-MA, which could inhibit the activity of autophagy, the decrease in expression of GLT-1 appeared to be exacerbated (Figure 4B). At the same time, uptake of $\left[{ }^{3} \mathrm{H}\right]$ glutamate by astrocytes infected with Htt-552 in the presence of rapamycin or 3-MA was determined. The result showed a recovery of glutamate uptake by astrocytes after treatment with rapamycin. In contrast, treatment with 3-MA slightly accelerated the decline of $\left[{ }^{3} \mathrm{H}\right]$ glutamate uptake in astrocytes expressing mutant $\mathrm{Htt}-552$, but the effect was not as significant (Figure 4C). 
A Con Null 18Q 100Q Con Null 18Q 100Q Con Null 18Q 100Q
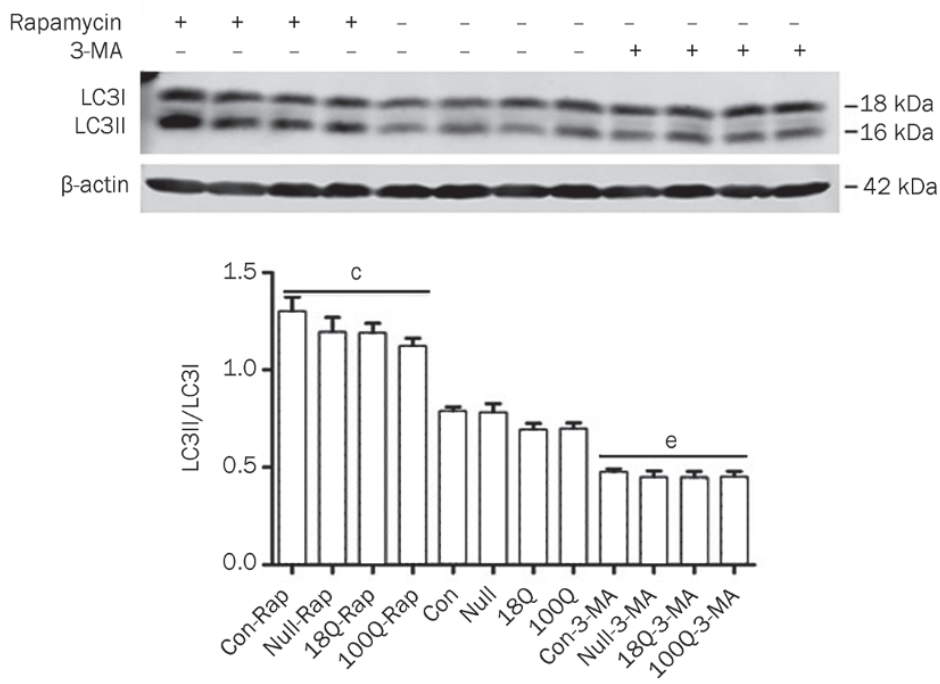

B Con Null 18Q 100 Q Con Null 18Q $100 Q$
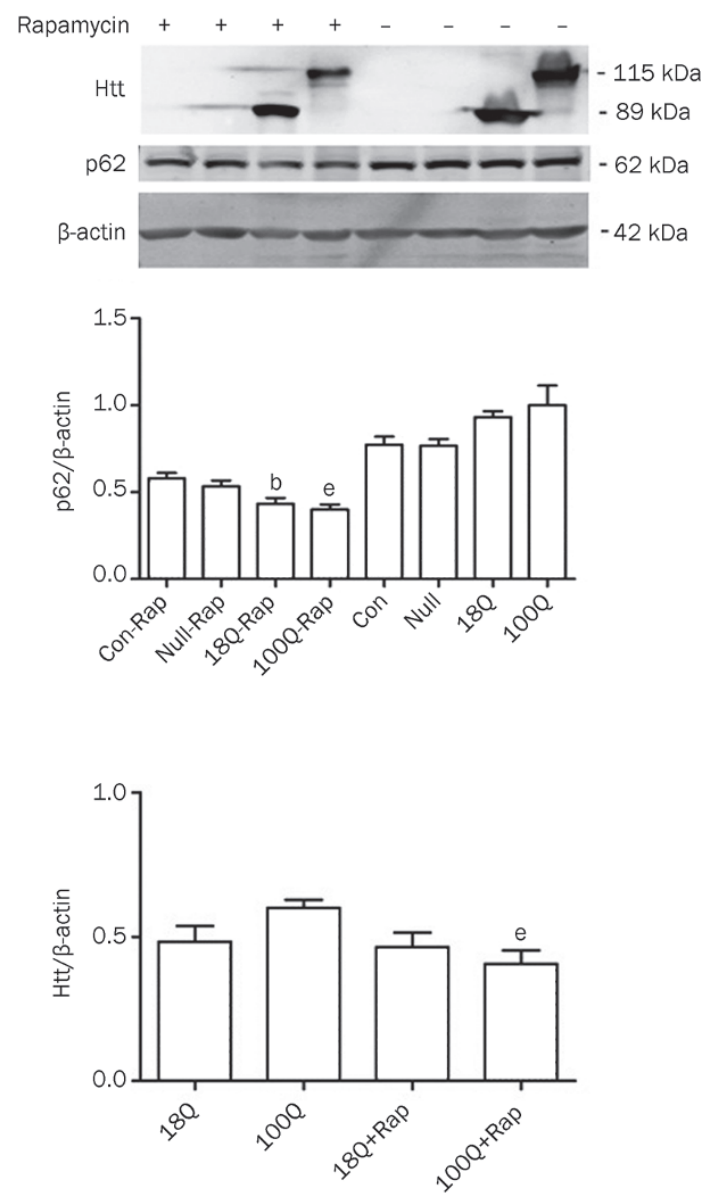

C Con Null $180100 Q$ Con Null $18 Q 100 Q$
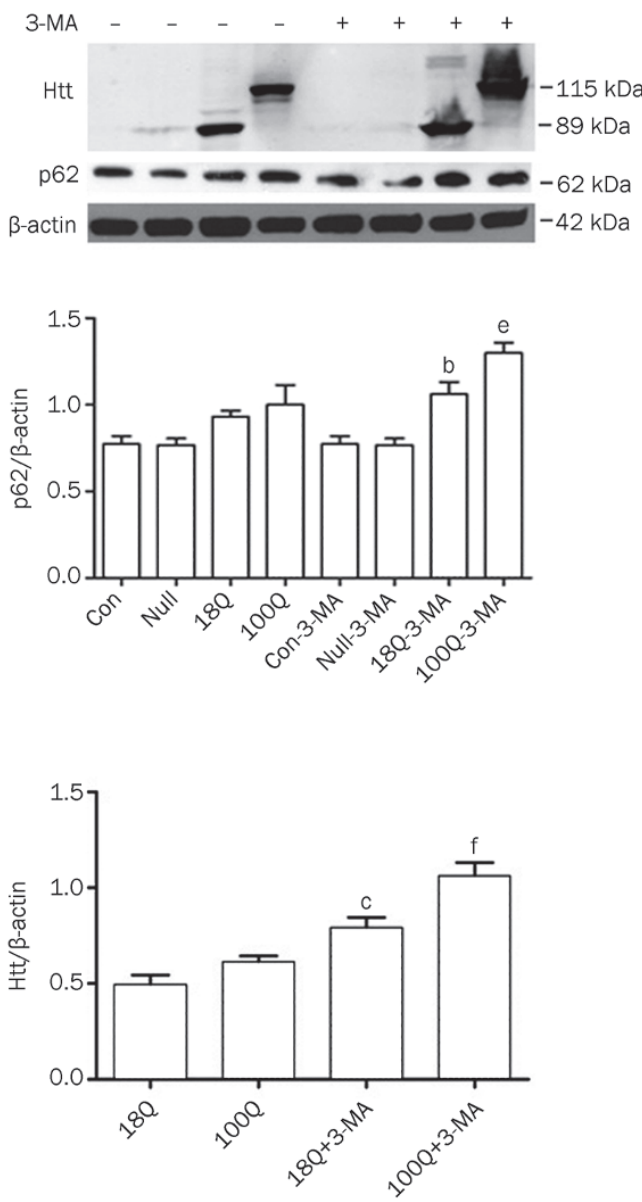

Figure 3. Mutant Htt-552 was reduced by enhanced autophagy. (A) Astrocytes were harvested after being infected for $72 \mathrm{~h}$ and treated with rapamycin $(0.2 \mu \mathrm{g} / \mathrm{mL})$ or 3-MA $(10 \mathrm{mmol} / \mathrm{L})$ for the last $24 \mathrm{~h}$. Western blotting results showed increased LC3II/LC3I with treatment of rapamycin and decreased LC3II/LC3I with the treatment of 3-MA ( ${ }^{\mathrm{C}} P<0.01$, rapamycin treatment vs control; ${ }^{\mathrm{e}} P<0.05$, 3-MA treatment vs control). (B) Western blotting results showed p62 expression level were decreased $\left({ }^{\mathrm{b} P}<0.05\right.$, 18Q-Rap vs 18Q, ${ }^{\mathrm{e}} \mathrm{P}<0.05$, 100Q-Rap vs 100Q) and the level of mutant Htt decreased with rapamycin treatment ( ${ }^{\mathrm{e}} \mathrm{P}<0.05,100 \mathrm{Q}-\mathrm{Rap}$ vs 100Q). (C) Western blotting results showed $\mathrm{P} 62$ expression level were increased $\left({ }^{\mathrm{b}} P<0.05,18 \mathrm{Q}-3-\mathrm{MA}\right.$ vs $18 Q ;{ }^{e} P<0.05,100 Q-3-M A$ vs 100Q) and the level of Htt increased ( ${ }^{C} P<0.01,18 Q-3-M A$ vs $18 Q ;{ }^{f} P<0.001,100 Q-3-M A$ vs $\left.100 Q\right)$ with 3-MA treatment. 
A
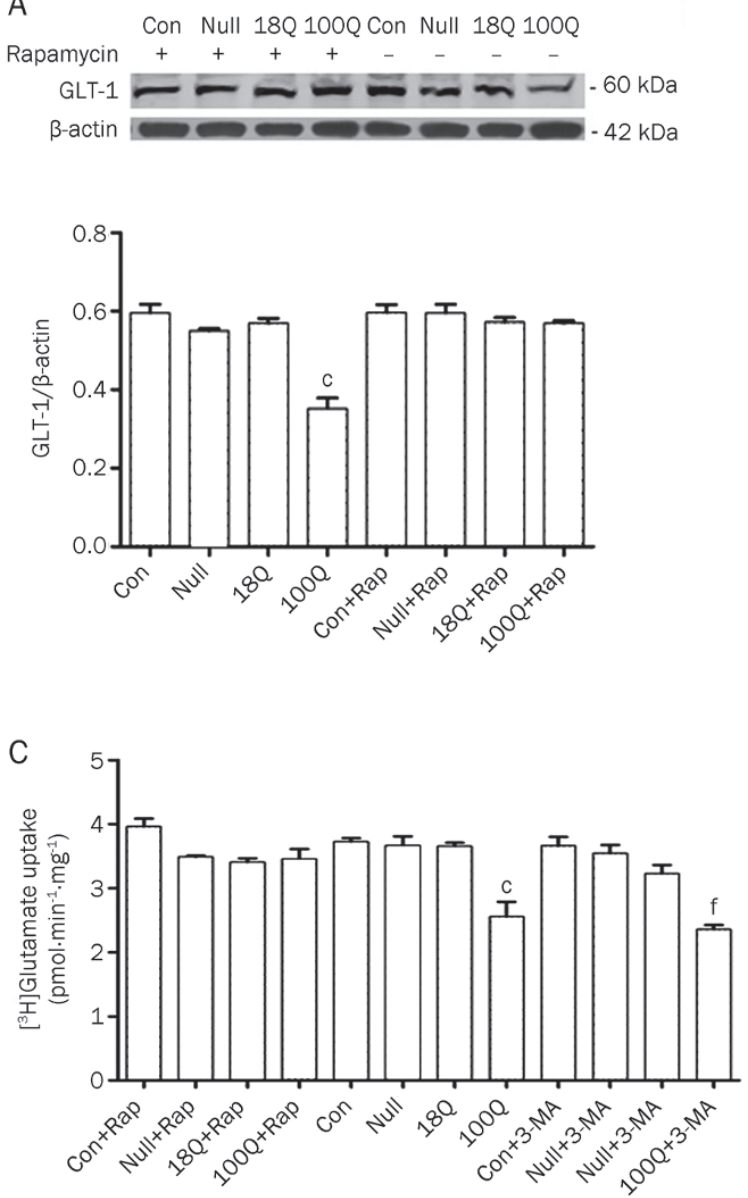

B
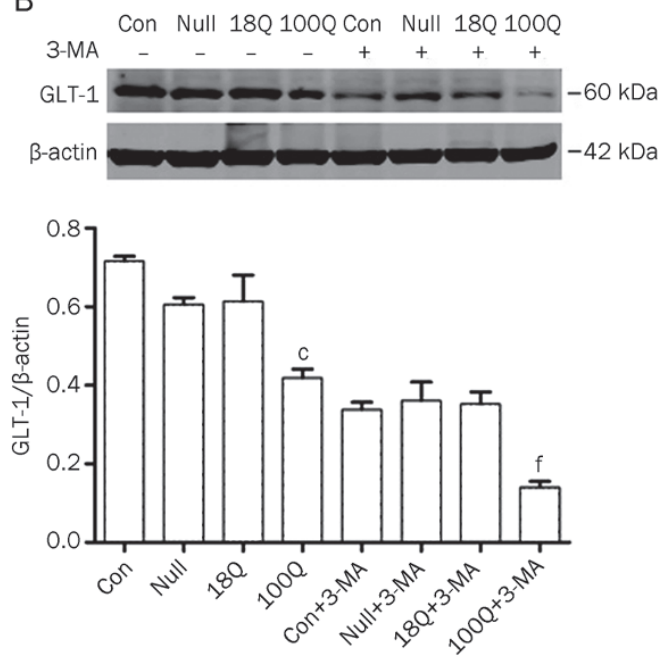

Figure 4. Recovered expression of GLT-1 and glutamate uptake by autophagy stimulator. The densities of respective protein bands in each group were analyzed with Sigma Scan Pro 5, and $\beta$-actin was used as a reference. All the results are shown as mean \pm SD $(n=3)$. (A) Astrocytes were harvested after being infected for $72 \mathrm{~h}$ and treated with rapamycin $(0.2 \mu \mathrm{g} / \mathrm{mL})$ during the last $24 \mathrm{~h}$. (A) Western blotting showing recovery of GLT-1 expression with stimulation of autophagy ( ${ }^{\mathrm{P}} \mathrm{P}<0.01,100 \mathrm{Q}-\mathrm{Rap}$ vs 100Q). (B) Astrocytes were harvested after being infected for $72 \mathrm{~h}$ and treated with 3-MA during the last $24 \mathrm{~h}$. Western blotting analysis showed decreased expression of GLT-1 with 3-MA treatment . ${ }^{\mathrm{f}} P<0.01$, 100Q-3-MA vs (con-3-MA, null-3-MA, 18Q-3$\mathrm{MA}) ;{ }^{\mathrm{c}} \mathrm{P}<0.01,100 \mathrm{Q}$ vs (con, null, 18Q). (C) Glutamate uptake was recovered by astrocytes expressing mutant Htt after treatment with rapamycin but not with 3-MA. Astrocytes were harvested after expression of Htt-552 for $72 \mathrm{~h}$ and treated with rapamycin $(0.2 \mu \mathrm{g} / \mathrm{mL})$ or 3-MA (10 mmol/L) for the last $24 \mathrm{~h}$, followed with incubation with [ $\left.{ }^{3} \mathrm{H}\right]$ glutamate for $15 \mathrm{~min}$ as described in the methods section. ${ }^{\mathrm{f}} \mathrm{P}<0.01,100 \mathrm{Q}-3-\mathrm{MA}$ vs (con-3-MA, null-3-MA, 18Q-3MA); ${ }^{\circ} P<0.01,100 Q$ vs (con, null, 18Q).

\section{Discussion}

In HD, mutant Htt is expressed in various types of cells, including neurons and non-neuronal cells, but it preferentially aggregates in striatal neurons and causes dysfunction in striatal neurons. There are two possible explanations for this phenomenon. First, compared with glial cells, lower and impaired neuronal ubiquitin-proteasome system activity, which plays a critical role in clearing misfolded proteins ${ }^{[18]}$, may account for the preferential accumulation of misfolded Htt in neurons as well as their selective vulnerability ${ }^{[19-21]}$. Second, the expression of mutant huntingtin in glial cells, which could clear excess excitatory neurotransmitters from extracellular space, contributed to neuronal excitotoxicity ${ }^{[2,5,22,23]}$.

In this paper, we intended to find out the effect of Htt-552, the fragment of Htt that exists in physiological and pathological conditions, on glutamate uptake in astrocytes. GLT-1 and
GLAST, two important types of glutamate transporters that are primarily expressed in astrocytes, were thought to be the predominant glutamate transporters and did the most work to clear extracellular excitatory glutamate ${ }^{[8]}$. Previous studies showed that mutant Htt expressed in astrocytes caused decreased GLT-1 both in HD mouse brains and in cultured glial cells, but there was no report about mutant Htt-552, which existed in the brains of HD patients. Consistent with prior research, we have provided direct evidence that mutant Htt-552 in glial cells reduced the expression of GLT-1 but not of GLAST and decreased glutamate uptake in astrocytes, which was mainly caused by decreased expression of GLT-1. Because there are Sp1-binding sites in the promoter of GLT-1 and the transcription of GLT-1 is Sp1-dependent ${ }^{[24,25]}$, previous reports of mutant Htt binding more Sp1 and reducing Sp1mediated GLT-1 expression in astrocytes may explain this 
phenomenon ${ }^{[10]}$.

Autophagy is thought to be involved in neurodegenerative diseases ${ }^{[26]}$, such as $\mathrm{HD}^{[27-29]}$, Parkinson's disease ${ }^{[30-33]}$, and Alzheimer's disease ${ }^{[34-36]}$. It is a major degradation pathway for long-lived misfolded proteins, such as mutant Htt in HD. In this study, we used rapamycin, an enhancer of autophagy, to activate autophagy. Although autophagy could be slightly activated by mutant $\mathrm{Htt}$, which might be the normal stress reaction of astrocytes, the effect of rapamycin would be more powerful. The present study showed that the expression and the glutamate uptake function of GLT-1 could be resumed after reducing mutant Htt-552 levels by stimulating autophagy. On the basis of our results, we postulated that a reduction in mutant Htt levels would increase the availability of Sp1 to the GLT-1 promoter and increase GLT-1 transcription back to initial levels. The dysfunction of the glutamate transporter GLT-1 caused by mutant Htt can be returned to normal after clearing mutant Htt. Our results give futher evidence for enhancing clearance of mutant Htt by autophagy may protect neurons survival by lower excitotoxicity in Huntington's disease $^{[37]}$.

\section{Acknowledgements}

We thank Prof Hai-yan LIU at Soochow University for providing help in the measurement of $\left[{ }^{3} \mathrm{H}\right]$-glutamate. This work is supported by grants from the National Natural Science Foundation of China (№ 30600197).

\section{Author contribution}

Dr Fang LIN designed the research; Lei-lei CHEN, Jun-chao WU, and Lin-hui WANG performed the research; Dr ZhengHong QIN, Dr Marian DIFIGILA, and Dr Jin WANG revised the paper.

\section{References}

1 Li H, Li S, Johnston H, Shelbourne P, Li X. Amino-terminal fragments of mutant huntingtin show selective accumulation in striatal neurons and synaptic toxicity, Nat Genet 2000; 25: 385-9.

2 Shin JY, Fang ZH, Yu ZX, Wang CE, Li SH, Li XJ. Expression of mutant huntingtin in glial cells contributes to neuronal excitotoxicity. J Cell Biol 2005; 171: 1001-12.

3 Gu X, Li C, Wei W, Lo V, Gong S, Li S, et al. Pathological cell-cell interactions elicited by a neuropathogenic form of mutant Huntingtin contribute to cortical pathogenesis in HD mice. Neuron 2005; 46: 433-44.

4 Gu X, Andre V, Cepeda C, Li S, Li X, Levine M, et al. Pathological cell-cell interactions are necessary for striatal pathogenesis in a conditional mouse model of Huntington's disease. Mol Neurodegener 2007; 2: 8.

5 Bradford J, Shin JY, Roberts M, Wang CE, Sheng G, Li S, et al. Mutant huntingtin in glial cells exacerbates neurological symptoms of Huntington disease mice. J Biol Chem 2010; 285: 10653-61.

6 Maragakis NJ, eeRothstein JD. Glutamate transporters in neurologic disease. Arch Neurol 2001; 58 : 365-70.

7 Faideau M, Kim J, Cormier K, Gilmore R, Welch M, Auregan G, et al. In vivo expression of polyglutamine-expanded huntingtin by mouse striatal astrocytes impairs glutamate transport: a correlation with Huntington's disease subjects. Hum Mol Genet 2010; 19: 3053-7.
8 Rothstein JD, Dykes-Hoberg M, Pardo CA, Bristol LA, Jin L, Kuncl $\mathrm{RW}$, et al. Knockout of glutamate transporters reveals a major role for astroglial transport in excitotoxicity and clearance of glutamate. Neuron 1996; 16: 675-86.

9 Su ZZ, Leszczyniecka M, Kang D, Sarkar D, Chao W, Volsky DJ, et al. Insights into glutamate transport regulation in human astrocytes: Cloning of the promoter for excitatory amino acid transporter 2 (EAAT2). Proc Natl Acad Sci U S A 2003; 100: 1955-60.

10 Bradford J, Shin JY, Roberts M, Wang CE, Li XJ, Li S. Expression of mutant huntingtin in mouse brain astrocytes causes age-dependent neurological symptoms. Proc Natl Acad Sci U S A 2009; 106:224805.

11 Berger Z, RavikumarB, Menzies FM, Oroz LG, Underwood BR, Pangalos $\mathrm{MN}$, et al. Rapamycin alleviates toxicity of different aggregate-prone proteins. Hum Mol Genet 2006; 15: 433-42.

12 Ravikumar B, Rubinsztein DC. Aggregate-prone proteins with polyglutamine and polyalanine expansions are degraded by autophagy. Hum Mol Genet 2002; 11: 1107-17.

13 Qin ZH, Qin YM, Kegel KB, Kazantsev A, Apostol BL, Thompson LM, et al. Autophagy regulates the processing of amino terminal huntingtin fragments. Hum Mol Genet 2003; 12: 3231-44.

14 Wang LH, Lin F, Wu JC, Qin ZH. High efficiency adenovirus-mediated expression of truncated $\mathrm{N}$-terminal huntingtin fragment (htt552) in primary rat astrocytes. Acta Biochim Biophys Sin 2009; 41: 325-34.

15 Kawahara K, Hosoya R, Sato H, Tanaka M, Nakajima T, Iwabuchi S. Selective blockade of astrocytic glutamate transporter GLT-1 with dihydrokainate prevents neuronal death during ouabain treatment of astrocyte/neuron cocultures. Glia 2002; 40: 337-49.

16 Tanida I, UenoT, Kominami E. LC3 and Autophagy. Methods Mol Biol 2008; 445: 77-88.

17 Pankiv S, Clausen TH, Lamark T, Brech A, Bruun JA, Outzen H, et al. p62/SQSTM1 binds directly to Atg8/LC3 to facilitate degradation of ubiquitinated protein aggregates by autophagy. J Biol Chem 2007; 282: 24131-45.

18 Li H, Li XJ, Li S. Clearance of mutant huntingtin. Autophagy 2010; 6: 663-4.

19 Tydlacka S, Wang CE, Wang X, Li S, Li XJ. Differential activities of the ubiquitin-proteasome system in neurons versus glia may account for the preferential accumulation of misfolded proteins in neurons. J Neurosci 2008; 28: 13285-95.

20 Wang J, Wang CE, Orr A, Tydlacka S, Li S, Li XJ. Impaired ubiquitinproteasome system activity in the synapses of Huntington's disease mice. J Cell Biol 2008; 180: 1177-89.

21 Bennett EJ, Shaler TA, Woodman B, Ryu KY, Zaitseva TS, Becker CH, et al. Global changes to the ubiquitin system in Huntington's disease. Nature 2007; 448: 704-8.

22 Brusilow WSA. Is Huntington's a glutamine storage disease? Neuroscientist 2006; 12: 300-4.

23 McBride JL, Ramaswamy S, Gasmi M, Bartus RT, Herzog CD, Brandon $E P$, et al. Viral delivery of glial cell line-derived neurotrophic factor improves behavior and protects striatal neurons in a mouse model of Huntington's disease. Proc Natl Acad Sci U S A 2006; 103: 9345-50.

24 Dunah AW, Jeong H, Griffin A, Kim YM, Standaert DG, Hersch SM, et al. Sp1 and TAFII130 transcriptional activity disrupted in early Huntington's disease. Science 2002; 296: 2238-43.

25 Li S, Cheng AL, Zhou H, Lam S, Rao M, Li H, et al. Interaction of Huntington disease protein with transcriptional activator Sp1. Mol Cell Biol 2002; 22: 1277-87.

26 Hara T, Nakamura K, Matsui M, Yamamoto A, Nakahara Y, SuzukiMigishima R, et al. Suppression of basal autophagy in neural cells causes neurodegenerative disease in mice. Nature 2006; 441: 
885-9.

27 Sarkar S, Rubinsztein DC. Huntington's disease: degradation of mutant huntingtin by autophagy. FEBS J 2008; 275: 4263-70.

28 Heng MY, Detloff PJ, Paulson HL, Albin RL. Early alterations of autophagy in Huntington disease-like mice. Autophagy 2010; 6: 1206-8.

29 Bauer PO, Nukina N. Enhanced degradation of mutant huntingtin by rho kinase inhibition is mediated through activation of proteasome and macroautophagy. Autophagy 2009; 5: 747-8.

30 Pan T, Rawal P, Wu Y, Xie W, Jankovic J, Le W. Rapamycin protects against rotenone-induced apoptosis through autophagy induction. Neuroscience 2009; 164: 541-51.

31 Dadakhujaev S, Noh HS, Jung EJ, Cha JY, Baek SM, Ha JH, et al. Autophagy protects the rotenone-induced cell death in alpha-synuclein overexpressing SH-SY5Y cells. Neurosci Lett 2009; 472: 47-52.

32 Winslow AR, Chen CW, Corrochano S, Acevedo-Arozena A, Gordon $\mathrm{DE}$, Peden $\mathrm{AA}$, et al. Rubinsztein DC, $\alpha$-Synuclein impairs macroautophagy: implications for Parkinson's disease. J Cell Biol 2010;
190: 1023-37.

33 Webb JL, Ravikumar B, Atkins J, Skepper JN, Rubinsztein DC. Alphasynuclein is degraded by both autophagy and the proteasome. J Biol Chem 2003; 278: 25009-13.

34 Yang DS, Lee JH, Nixon RA. Monitoring autophagy in Alzheimer's disease and related neurodegenerative diseases. Methods Enzymol 2009; 453: 111-44.

35 Boland B, Kumar A, Lee S, Platt FM, Wegiel J, Yu WH, et al. Autophagy induction and autophagosome clearance in neurons: relationship to autophagic pathology in Alzheimer's disease. J Neurosci 2008; 28 : 6926-37.

36 Nixon RA, Yang DS. Autophagy failure in Alzheimer's disease-locating the primary defect. Neurobiol Dis 2011; 43: 38-45.

37 Ravikumar B, Vacher C, Berger Z, Davies JE, Luo S, Oroz LG, et al. Inhibition of mTOR induces autophagy and reduces toxicity of polyglutamine expansions in fly and mouse models of Huntington disease. Nat Genet 2004; 36: 585-95. 